\title{
Dinámica de opciones de grado de programas en modalidad presencial
}

Options of degree's dynamics in face to face university programs 


\title{
Dinámica de opciones de grado de programas en modalidad presencial $^{1}$
}

\section{Options of degree's dynamics in face to face university programs}

\author{
Carlos Alberto Gómez Cano², Verenice Sánchez Castillo ${ }^{3}$ \& Paola Andrea Hernández S. ${ }^{4}$ \\ Universidad de la Amazonia, Florencia, Caquetá
}

Citación del artículo: Gómez, C., Sánchez, V. \& Hernández, P. (2016). Dinámica de opciones de grado de los programas en modalidad presencial de la Facultad de Ciencias Contables, Económicas y Administrativas (FCCEA) de la Universidad de la Amazonia (2013-2015). I+D Revista de Investigaciones, 8(2), pp. 73 - 82

\begin{abstract}
Resumen
La autonomía universitaria ha posibilitado la generación de condiciones propias para cada institución de educación superior (IES) según su contexto, requerimientos y condiciones. En tal sentido, las universidades han desarrollado su propia legislación en temas académicos, como las opciones de grado, que, para el caso específico de la Universidad de la Amazonia se han condensado en el Acuerdo 021 de 2009 del Consejo Académico. La presente investigación acude al paradigma de investigación crítico social, tipo de investigación descriptiva y al enfoque de investigación mixto, en razón a que los datos son cuantitativos y cualitativos. En tal sentido, fruto del proceso de investigación, se caracterizó la dinámica de las opciones de grado de la Facultad de Ciencias Contables, Económicas y Administrativas (FCCEA) en el periodo de estudio, la percepción docente respecto a esta y los retos que debe afrontar la FCCEA en el actual contexto de la educación superior en Colombia. El balance final es que la transformación de la dinámica de las opciones de grado exige la intervención activa de todos los agentes del proceso educativo, es decir, un trabajo sinérgico entre la dirección administrativa, los docentes y los estudiantes.
\end{abstract}

Palabras clave: investigación, estudiantes, desarrollos, docentes.

\section{Abstract}

University autonomy has enabled the generation of each IES own conditions, depending on the context, requirements and conditions. In this sense, universities have developed their own legislation on academic subjects, such as degree options, which in the specific case of the University of the Amazon have been condensed in the Agreement 021 of

1. Artículo de investigación crítico-social, de enfoque cualitativo y cuantitativo, resultado del proyecto de investigación "Caracterización de las opciones de grado de la Universidad de la Amazonia", perteneciente al área Contable y Administrativa, desarrollado en el grupo de investigación de la Facultad de Ciencias Contables, Económicas y Administrativas FCCEA y financiado por la Universidad de la Amazonia de la ciudad de Florencia (Colombia). Dirección: Calle 17 Diagonal 17 con Carrera 3F, barrio Porvenir, PBX: 4358786. Fecha de inicio: junio 2015, fecha de terminación: junio

2016.

2. Contador público, Universidad de la Amazonia. Administrador público, Escuela Superior de Administración Pública. Especialista en Pedagogía, Universidad de la Amazonia y Especialista en Gestión Pública, Escuela Superior de Administración Pública. Docente-investigador del Grupo de Estudio de Futuro en el Mundo Amazónico (GEMA). Universidad de la Amazonia de la ciudad de Florencia (Colombia). Dirección: Calle 17 Diagonal 17 con Carrera 3F, barrio Porvenir, PBX: 4358786. car.gomez@udla.com

3. Ingeniera agroecóloga, Universidad de la Amazonia. MSc. en Ambiente y Desarrollo, Universidad Iberoamericana Puebla. Docente-investigadora del Grupo de Investigación en Agroecología y Desarrollo Rural (GIADER). Universidad de la Amazonia de la ciudad de Florencia (Colombia). Dirección: Calle 17 Diagonal 17 con Carrera 3F, barrio Porvenir, PBX: 4358786. ve.sanchez@udla.edu.co

4. Estudiante del Programa de Contaduría Pública de la Universidad de la Amazonia, integrante del Semillero Facultad de Ciencias Contables, Económicas y Administrativas (FCCEA). Universidad de la Amazonia de la ciudad de Florencia (Colombia). Dirección: Calle 17 Diagonal 17 con

Carrera 3F, barrio Porvenir, PBX: 4358786. Correo electrónico institucional: p.hernandez@udla.edu.co 
2009 of the Academic Council. This research comes to the paradigm of critical social research, descriptive type of research and research approach used was mixed, due to the nature of the data they are quantitative and qualitative. In this sense, the result of the research process, the dynamics of options degree of the Faculty in the study period, the teacher insight into this and the challenges facing the FCCEA in the current context of higher education was obtained in Colombia, leaving a final balance that the transformation of the dynamics of degree options requires the active involvement of all stakeholders in the educational process, so, a synergistic work between the administrative management, teachers and students.

Keywords: research, students, academic development, teachers.

\section{La educación superior y su autonomía}

La educación es considerada como una fuerza transformadora de la sociedad (Unesco, 2015), pues el perfeccionamiento de competencias académicas y científicas genera mejores escenarios para el desarrollo de los seres humanos (Ospina, 2008). En el caso de Colombia, la educación ha sido catalogada como un derecho fundamental de los niños en el Artículo 44, un derecho de la persona y un servicio público en el Artículo 67, todo ello en la Constitución Política de Colombia (Gómez, 2015).

En el caso específico de la educación superior, lbáñez (1994, p. 1) sostiene que esta tiene como objetivo el desarrollo de capacidades, habilidades y actitudes para la transformación de la sociedad; además, afirma que la educación superior es "la formación de profesionales competentes; individuos que resuelvan creativamente, es decir, de manera novedosa, eficiente y eficaz, problemas sociales". Misas (2004), por su parte, afirma que la educación superior tiene un rol protagónico en el desarrollo económico, social y político; además, hace hincapié en la responsabilidad que atañe a las universidades en la trasformación de los escenarios sociales.

Así las cosas, las universidades deben, entre otras cosas, responder a las necesidades específicas de su contexto social, por lo que pensar en procesos de estandarización y homogenización en la educación superior es algo poco procedente. En concordancia con lo anterior, la Constitución Política de Colombia (CPC), a través de su Artículo 69, establece que las universidades, en su calidad de entes autónomos, pueden darse sus directivas y regirse por sus propios estatutos, de acuerdo con la ley.

Unido a lo anterior, la Ley 30 de 1992 establece, en su artículo tercero, que "El Estado, de conformidad con la Constitución Política de Colombia y con la presente Ley, garantiza la autonomía universitaria, y vela por la calidad del servicio educativo a través del ejercicio de su suprema inspección y vigilancia de la Educación Superior" (Congreso de la República de Colombia, 1992), articulado que ratifica la importancia de la autonomía universitaria como una herramienta para legislar sobre contextos específicos y tomar acciones particulares, según los escenarios de cada institución.

Finalmente, es importante precisar que la autonomía universitaria no es sinónimo de poder absoluto, sino la posibilidad de organizar las universidades de la mejor manera para cumplir su misión social, en el marco legal exigido por la CPC y demás disposiciones (Villegas, s. f.), ventaja que aprovechada de buena manera, permitirá a cada institución identificar sus necesidades, atenderlas, generar nuevos procesos y reingenierías, buscando atender las dinámicas de su territorio.

Las opciones de grado en la Universidad de la Amazonia Como una muestra de la autonomía universitaria, cada institución de educación superior ha generado dinámicas en torno a sus procesos académicos, ello explicaría la diversidad existente en los currículos, metodologías, trámites académicos, modalidades de formación y formas de intervención en el territorio en las dimensiones de investigación, extensión y proyección social.

En el caso específico de las opciones de grado, las cuales, según la Corporación Universitaria Minuto de Dios (2014), son propuestas académicas que buscan fortalecer competencias del ser y generar espacios para reforzar las capacidades argumentativas, críticas, creativas e innovadoras, y profundizar en sus competencias profesionales en contexto, cada institución de educación superior ha creado sus propias modalidades, utilizando el principio de autonomía universitaria y los fundamentos filosóficos que las cimienta.

Para la Universidad de la Amazonia, entidad oficial del orden nacional, transformada a través de las Ley 60 de 1982, el Consejo Superior Universitario, a través del Artículo 64 del Acuerdo 09 de 2007 reglamentó las opciones de grado para los estudiantes de pregrado, decisión normalizada a través del Acuerdo 021 de 2009 del Consejo Académico, donde se establecen las siguientes opciones de grado: 
- Pasantías o servicios a la comunidad. La pasantía o servicio a la comunidad es un proceso sistemático que realiza individualmente un estudiante en una organización legalmente constituida, donde se ponen en práctica los conocimientos adquiridos durante su carrera para desarrollar una actividad que genere un impacto específico, en correspondencia con los propósitos de formación de su programa académico.

- Participación significativa y de relevancia en proyectos de investigación o de gestión, debidamente certificados por la Vicerrectoría de Investigaciones, según sea el caso. Se entiende como la ejecución de actividades significativas que desarrolla el estudiante en proyectos relevantes de investigación o de gestión, debidamente certificados por la Vicerrectoría de Investigaciones. Su objetivo principal es incentivar la formación en investigación o gestión a los estudiantes de pregrado.

- Trabajo de grado. Se entiende por trabajo de grado el trabajo de investigación elaborado por el estudiante, que le permita desarrollar habilidades para identificar y formular problemas, y plantear o implementar alternativas de solución posibles mediante la utilización de la lógica del accionar científico.

- Monografía. Se denomina monografía a los textos de trama argumentativa y función informativa que organizan, en forma analítica y crítica, datos sobre un tema compilados de diferentes fuentes.

- Judicatura. Se entiende como el ejercicio de las funciones jurídicas de los estudiantes de Derecho que han cursado y aprobado todos los cursos de formación del plan de estudios y se desarrollará de conformidad con la normatividad nacional vigente.

- Exámenes preparatorios. Son exámenes orales, presentados de manera personal ante un jurado compuesto por dos docentes que posean una experiencia docente universitaria mínima de dos años, con formación de pregrado y postgrado en el área que se evalúa.

- Seminarios de profundización. Es el estudio de temáticas sinérgicamente relacionadas, orientadas a perfeccionar los conocimientos del estudiante en áreas propias de su formación disciplinar.

- Haber publicado un artículo en una revista indexada. Es la participación del estudiante como autor principal o coautor de un artículo publicado o aceptado para publicación en una revista indexada del orden nacional o internacional.
- Semestre avanzado. Figura que permite a un estudiante de un programa de pregrado que ha terminado la totalidad de los cursos previstos en el plan de estudios, tomar todos los créditos correspondientes a un primer semestre de un programa de posgrado propio de esta institución. Se exige promedio académico acumulado igual o superior a (3.8).

Es importante precisar que para la FCCEA la judicatura no aplica, pues esta figura es exclusiva para los estudiantes de la Facultad de Derecho y Ciencias Políticas. Las otras ocho opciones de grado están disponibles para quienes aspiren a obtener su título profesional.

En tal sentido, el presente manuscrito busca realizar un análisis crítico de la dinámica de las opciones de grado desarrolladas por los estudiantes de los programas en modalidad presencial de la Facultad de Ciencias Contables, Económicas y Administrativas (FCCEA) de la Universidad de la Amazonia durante los años 2013 2015, donde se caracterizará la participación de cada una de las modalidades disponibles, la percepción de los docentes y líderes de investigación, y, finalmente, se hace una reflexión acerca de los retos que asumiría la FCCEA respecto a sus dinámicas académicas.

\section{Materiales y métodos}

\section{Localización}

La pesquisa se llevó a cabo en la Universidad de la Amazonia, localizada en el municipio de Florencia, Departamento del Caquetá, el cual está localizado a los $1^{\prime} 37^{\prime} 03^{\prime \prime}$ de latitud norte y $75^{\prime} 37^{\prime} 03^{\prime \prime}$ de longitud oeste, tiene una altura promedio de 242 metros sobre el nivel del mar, una temperatura media de $24.8^{\prime} \mathrm{C}^{\circ}$ y una precipitación media anual $3840 \mathrm{~mm}$. Florencia dista de la capital de la República 563 km (Alcaldía de Florencia, 2016). La población participante del estudio fueron los docentes e investigadores de la Facultad de Ciencias Contables, Económicas y Administrativas (FACCEA) de la mencionada universidad, quienes han estado en directa relación con los procesos misionales de esta alma mater.

\section{El enfoque metodológico}

El paradigma de investigación que guio la presente investigación fue el crítico social, pues con el presente trabajo se busca promover las transformaciones sociales y dar respuesta a un problema específico, el cual está presente en una comunidad (Alvarado \& García, 2008), en este caso la académica, con la respectiva participación de sus miembros. Para estos autores, el paradigma socio-crítico se fundamenta en la crítica social con un 
marcado carácter autorreflexivo, el cual sostiene que el conocimiento es una construcción en torno a intereses y necesidades que parten de los grupos.

La investigación es descriptiva, pues involucra la descripción, el registro, el análisis y la interpretación de la naturaleza actual, así como el proceso de los fenómenos (Tamayo \& Tamayo, 2002). Debido al tipo de datos producidos, los instrumentos y las técnicas para el levantamiento de la información, la investigación tuvo un enfoque mixto -cualitativo y cuantitativo-.

\section{El método}

Análisis de las opciones de grado disponibles en la Uniamazonia. Para esta sección se acudió a la técnica de análisis documental y arqueo de archivo. En ella, se identificó la documentación y normatividad interna que regula las opciones de grado en la Uniamazonia. De igual forma, se abordaron los procedimientos y requisitos en cada una de ellas. La sistematización de la información, se hizo a partir de plantillas de doble entrada donde se colocó la norma, la descripción y la síntesis del hallazgo.

Identificación de las opciones de grado elegidas por los estudiantes de la FCCEA. Posteriormente, empleando también la técnica de arqueo de archivo, pero esta vez interno, dentro de la FCCEA, se rastrearon las diferentes opciones de grado por las cuales había optado cada uno de los egresados de los programas de Contaduría Pública y Administración de Empresas durante el periodo 20132015.

Esta información fue puesta en matrices donde se enlistaron cada una de las opciones de grado disponibles; posteriormente, se determinó el número de opciones tomadas en cada caso por programa y por año. La información, identificada inicialmente en números, fue llevada a porcentajes y se realizaron los respectivos gráficos para el análisis. De esta manera, las variables de análisis fueron: año, programa y opciones de grado elegidas.

Caracterización de la percepción social de los investigadores y docentes de la FACCEA acerca de las opciones de grado elegidas por los estudiantes. El panorama cuantitativo en relación a las opciones de grado de la FCCEA fue socializado con docentes, investigadores y directivas académicas de la misma facultad, a quienes una vez conocieron estos resultados, se les realizó una entrevista abierta donde se abordaron variables de percepción acerca de prioridad de los seminarios de profundización como opción de grado, limitantes para el proceso de investigación, estrategias para el fomento de la investigación.

Las entrevistas fueron grabadas, previa autorización de los entrevistados, transcritas y llevadas al programa de procesamiento de datos cualitativos Atlas Ti. En él se seleccionaron las frases de interés, se establecieron códigos y sus respectivas relaciones, lo cual permitió generar las redes de trabajo. A partir de esto se trianguló la información.

\section{Resultados y discusión}

\section{Dinámica de las opciones en la FCCEA}

La Facultad de Ciencias Contables, Económicas y Administrativas (FCCEA) es una de las unidades académicas con las que se forjó la Universidad de la Amazonia, situación que le genera una representación importante en la población estudiantil. Ratificando lo anterior, se tiene que para la vigencia 2015, según la División de Admisiones, Registro y Control Académico, la FCCEA representaba poco más del $25 \%$ de la población estudiantil de la Universidad de la Amazonia, situación que es atribuible a que sus programas se ofrecen en jornada diurna, nocturna y modalidad distancia.

En cuanto a las opciones de grado, la FCCEA graduó a 477 profesionales de la modalidad presencial, que representan el $32 \%$ de total de los graduados de la Universidad de la Amazonia, los cuales desarrollaron las opciones de grado que se describen a continuación:

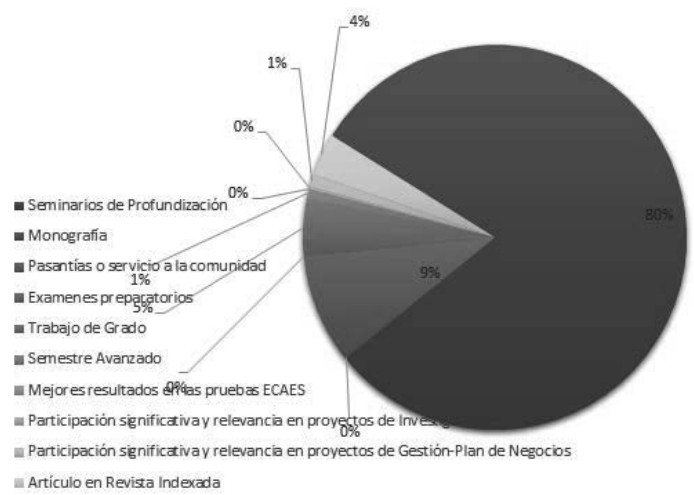

Figura 1.

Opciones de grado FCCEA (2013-2015). Programas modalidad presencial. Fuente: Archivos institucionales. Cálculos propios.

Talycomolodetallalafigura, existeuna marcadatendencia por la realización de seminarios de profundización, pues 383 estudiantes, quienes representan el $80 \%$ del total de los graduados en el periodo de estudio, acudieron a esta opción. Esta situación es perfectamente válida desde 
una perspectiva institucional, pues está contemplada entre las opciones de grado; sin embargo, siguiendo a Restrepo (s. f.) y Ruiz (2010), se está desatendiendo una de las funciones básicas de la Universidad, la cual está relacionada con la generación de nuevo conocimiento a través de procesos de investigación científica, tecnológica, humanística y social, pues los seminarios son espacios de actualización y perfeccionamiento en los cuales no se da un proceso riguroso de investigación. La segunda opción en términos de participación es la pasantía o servicio a la comunidad, la cual se convierte en una ventana para generar procesos de reciprocidad e intervención en la sociedad mediante los futuros profesionales. Su representación está en 9\%, 44 estudiantes, quienes a través de su ejercicio fortalecen la proyección social, uno de los ejes estratégicos de la Universidad. Sin embargo, esta opción de grado no es la única forma de intervenir en los nichos sociales, pues, según Sierra $(2004$, p. 1) “Las universidades son las más llamadas a generar investigación, por cuanto se supone que allí están las personas más idóneas y experimentadas en su ciencia o disciplina específica para compartir el acervo de saberes, guiar en la búsqueda a quienes apenas comienzan la formación en la educación superior y servir como apoyo al desarrollo de la sociedad."

Esta situación conduce reflexionar en torno a la importancia de promover la investigación, no solo como un ejercicio académico de perfeccionamiento, sino como una herramienta de intervención social y de mejoramiento de las condiciones de los territorios. Es decir, la investigación puede ser considerada como una sólida herramienta para el desarrollo integral de los ejes misionales de la Universidad (Mejía, s.f.).

Ahora bien, puesto que conviene realizar un análisis detallado del comportamiento de las opciones de grado en cada uno de los programas a fin de generar mayor elementos para la discusión, se auscultaron los archivos institucionales de los dos programas académicos de la FCCEA en modalidad presencial, es decir, Contaduría Pública y Administración de Empresas, encontrándose los siguientes resultados:

Al detallar la Tabla 1, llama la atención que no existan en el programa de Contaduría Pública estudiantes con artículos científicos publicados, pues todos están concentrados en el programa de Administración de Empresas. Asimismo, es incipiente la participación en los proyectos relevantes de investigación, figuras que podrían generar un mayor acercamiento al desarrollo de competencias en investigación en los estudiantes. Por su parte, la participación de los trabajos de grado, que pueden ser otro camino para acercarse a los desarrollos
Tabla 1

Comportamiento de las opciones de grados de los programas de modalidad presencial de FCCEA

\begin{tabular}{|c|c|c|c|c|c|c|}
\hline \multirow[t]{2}{*}{ Opciones de grado } & \multirow{2}{*}{$\begin{array}{l}\text { Contaduría } \\
\text { Pública }\end{array}$} & \multirow{2}{*}{$\begin{array}{l}\text { Administración } \\
\text { de Empresas }\end{array}$} & \multirow[t]{2}{*}{ Total } & \multicolumn{2}{|c|}{$\begin{array}{l}\text { Porcentaje } \\
\text { del Programa }\end{array}$} & \multirow{2}{*}{$\begin{array}{l}\text { Porcent } \\
\text { aje de la } \\
\text { Facultad }\end{array}$} \\
\hline & & & & PCP & PAE & \\
\hline $\begin{array}{l}\text { Seminarios de } \\
\text { profundización }\end{array}$ & 2651 & 18 & 3838 & $2 \% 7$ & $8 \%$ & $80 \%$ \\
\hline Monografía 0 & 0 & 0 & 0 & $\%$ & $0 \%$ & $0 \%$ \\
\hline $\begin{array}{l}\text { Pasantías o servicio a la } \\
\text { comunidad }\end{array}$ & 431 & 4 & 4 & $13 \%$ & $1 \%$ & $9 \%$ \\
\hline Exámenes preparatorios & 0 & 0 & 0 & $0 \% 0$ & $\%$ & $0 \%$ \\
\hline Trabajo de grado 1 & 1 & 122 & 3 & $3 \% 8$ & $\%$ & $5 \%$ \\
\hline Semestre avanzado 3 & 1 & 4 & 1 & $\%$ & $1 \%$ & $1 \%$ \\
\hline $\begin{array}{l}\text { Mejores resultados en las } \\
\text { pruebas Ecaes }\end{array}$ & 0 & 0 & 0 & $0 \% 0$ & $\%$ & $0 \%$ \\
\hline $\begin{array}{l}\text { Participación significativa y } \\
\text { relevancia en proyectos de } \\
\text { Investigación }\end{array}$ & 1 & 0 & 1 & $0 \% 0$ & $\%$ & $0 \%$ \\
\hline $\begin{array}{l}\text { Participación significativa y } \\
\text { relevancia en proyectos de } \\
\text { gestión-plan de negocios }\end{array}$ & 2 & 3 & 5 & $1 \% 2$ & $\%$ & $1 \%$ \\
\hline $\begin{array}{l}\text { Artículo en revista } \\
\text { indexada }\end{array}$ & 0 & 171 & 7 & $0 \% 1$ & $1 \%$ & $4 \%$ \\
\hline TOTAL & 3251 & 52 & 477 & $100 \%$ & $100 \%$ & $100 \%$ \\
\hline
\end{tabular}

\section{Fuente}

Archivos institucionales. Cálculos propios

investigativos, no supera el $5 \%$; incluso, opciones tan llamativas como la realización de un posgrado como semestre avanzado o la elaboración de un plan de negocios en compañía de la unidad de emprendimiento, alcanzan $\tan$ solo el $1 \%$, lo que es realmente cuestionable en una facultad con disciplinas contables y administrativas, además, con la mayor cantidad de posgrados de la institución.

Sobre el particular, Silva, Rodríguez, Gonzales y Sarmiento (2008), consideran que para su caso de estudio - Facultad de Ciencias Económicas de la Universidad Militar Nueva Granada - existen factores endógenos y exógenos que limitan la participación de los estudiantes en procesos de investigación, por lo que es imperioso involucrar al colectivo docente en las dinámicas de opciones de grado, para que ellos, como actor estratégico del proceso de formación, impulsen el desarrollo de la investigación en el estudiantado (Ministerio de Educación Nacional [MEN], 2009). Una de las estrategias que exponen los autores está relacionada con la inclusión de la investigación como eje transversal en el currículo, garantizando de esta manera un acercamiento constante a las dinámicas investigativas.

Así las cosas, la transformación de las opciones de grado se dará de manera secundaria una vez se generen nuevas dinámicas investigativas en la Facultad, es decir, que en la medida en que se incorporen mayores exigencias respecto de los procesos educativos, se generarán nuevas dinámicas en los futuros profesionales. En tal sentido, 
Robalino (2005) afirma que los cambios educativos solo serán posibles por la intervención de los docentes, lo que invita a una reflexión, siguiendo a Maciel de Oliveira (2005), sobre la importancia de formar constantemente a los docentes para empoderarlos de su rol protagónico en el sistema educativo.

\section{Percepción docente respecto a las opciones de grado}

Como se mencionó con anterioridad, los docentes son agentes protagónicos en el proceso educativo, pues sus acciones inciden, directa o indirectamente, sobre los educandos (Prieto, 2008); además, su accionar debe dar cuenta de los objetivos institucionales que, para el caso de la educación superior, giran en torno a actividades de docencia, investigación y proyección social (Alfonso de Silvero, 2012).

Así las cosas, se realizaron entrevistas a docentes que lideran procesos de investigación dentro de los programas objeto de estudio, identificando las siguientes categorías de análisis.

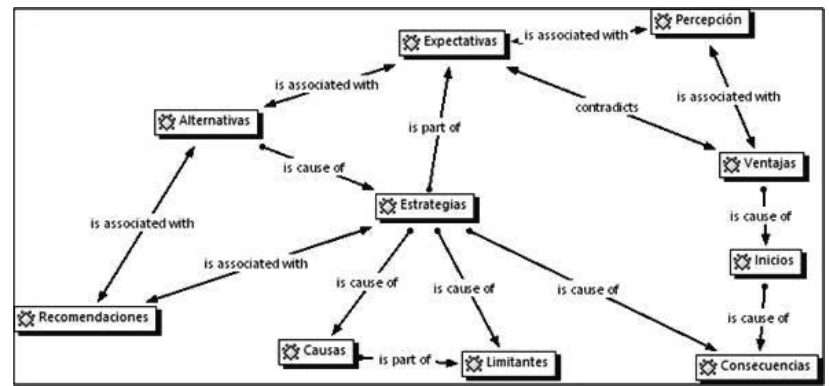

Figura 2.

Percepción docente respecto a las opciones de grado. Fuente: Los autores.

Tal y como lo detalla la Figura 2, a partir de las entrevistas a los docentes se generaron diez categorías de análisis en relación con su percepción sobre las opciones de grado de la FCCEA. Los docentes señalan que el auge de los seminarios de profundización sobre las demás opciones de grado se debe a las ventajas y facilidades para su desarrollo, toda vez que solo se requiere cumplir con 160 horas de trabajo presencial y cubrir la carga monetaria exigida por la Universidad, por lo que se convierten en una opción sencilla y rápida respecto de las otras opciones de grado, tales como tesis, participación en proyectos de investigación o escritura de artículos científicos.

Este escenario contradice las expectativas de los educadores, quienes consideran que se está dejando atrás la investigación, siendo esta un eje estratégico de la Universidad. Además, afirman que han implementado estrategias de mejora, tales como motivación por la lectura crítica, creación de espacios de debate y desarrollo académico y una constante promoción de los semilleros de investigación con los que cuenta la Facultad. Lo anterior haciendo hincapié en los beneficios con que cuentan los jóvenes investigadores.

Asimismo, durante el desarrollo de las entrevistas, los docentes propusieron las siguientes recomendaciones $y$ alternativas:

Trabajos integrados. Se requiere realizar trabajos conjuntos, que permitan a los estudiantes integrar todo su proceso educativo, especialmente con los docentes que lideran las áreas de redacción y comunicación a fin de desarrollar competencias que permitan mejores desarrollos en el campo de la investigación e indicadores de calidad (Antúnez, 1999).

Formación en investigación. Es indispensable desarrollar seminarios permanentes de investigación para docentes y estudiantes, con el propósito de fomentar los espacios de trabajo y desarrollo investigativo. En palabras de Tovar, Gálvez y Cárdenas (2012), se deben generar escenarios de formación estratégica para cada contexto educativo.

Estas acciones, a juicio de los entrevistados, pueden contrarrestar las causas y limitantes que han consolidado a los seminarios de profundización como la opción de mayor preferencia en la Facultad, pues los mencionados escenarios permitirán que los estudiantes desarrollen su opción de grado durante su trasegar académico, especialmente aquellos de la jornada nocturna, quienes por sus ocupaciones laborales dedican poco tiempo a estos asuntos.

Finalmente, los participantes expresan requerir mayor apoyo de la institución para la flexibilización de los trámites administrativos -adquisición de bienes y servicios propios de los procesos de investigación-, mejores condiciones en la labor docente, como un mayor reconocimiento de los tiempos para hacer investigación y mayor acceso a recursos para el desarrollo los proyectos.

\section{Los retos de la FCCEA en sus procesos académicos}

Melo, Ramos y Hernández (2014) afirman que el éxito de la educación superior en Colombia depende de una gama amplia de factores, entre los cuales se encuentran la calidad del personal docente, los incentivos a la investigación y la motivación de los estudiantes. Además, sostienen que la difíciles condiciones de vinculación laboral y la poca formación doctoral "pueden estar 
afectando las actividades de investigación, la formación académica de los estudiantes y en general los resultados del sistema educativo." (p. 20).

Sobre el particular, Misas (2004, p. 9), sostiene que "es urgente que la universidad construya una visión de futuro, que permita al sistema de educación superior orientar sus esfuerzos en el desarrollo y consolidación de los programas de formación e investigación que se consideren estratégicos para alcanzar las metas que se fije la sociedad". En tal sentido, y dado que las realidades de la Universidad de la Amazonia y de la FCCEA no son ajenas a las dinámicas nacionales, se tipifican algunos retos para los procesos académicos, entre los que se pueden considerar:

Generación de una cultura de investigación: Sierra , Rojas y López (2009), sostienen que uno de los grandes retos de los educadores en la actual sociedad es desprenderse del paradigma de "dictar clase" para dar paso una nuevas formas de interacción con el estudiantado, donde la investigación nazca como un espacio de reflexión y desarrollo complementario; es decir, la educación se debe contemplar como una realidad social para la transformación de las culturas, donde la investigación es la principal estrategia de intervención (Universidad de la Salle, s. f.).

Mejoramiento de las condiciones de los docentes. Tal y como se ha mencionado con anterioridad, es importante que los docentes intervengan en los procesos de investigación y transformación de los escenarios académicos; sin embargo, en esta tarea es imprescindible mejorar sus condiciones, pues según Cornejo (2009) existen grandes contrastes entre las exigencias laborales y las condiciones de trabajo del profesorado, lo que podría dar cuenta de la poca participación en procesos vitales como la investigación. Esta situación no es ajena para las autoridades del país, pues el MEN (2009) considera que ofrecer condiciones estables y dignas generará retención de los maestros y mejores resultados en el sistema educativo.

Mejoramiento de los canales de comunicación institucional. Barragán y Patiño (2013) sostienen que en Colombia la rigidez de los sistemas administrativos de las IES contribuye a ampliar la barrera entre la universidad y los estudiantes, pues no existe una política de inmersión a la vida universitaria para estos últimos. Esta realidad, para nuestro caso específico de estudio, se materializa en la poca información que obtienen los estudiantes durante su trasegar académico acerca de las opciones de grado, impidiendo que estas se desarrollen de manera paralela con los compromisos de aula, obligando, de cierta manera a tomar opciones más rápidas, como la de los seminarios de profundización.

\section{Conclusiones}

Uno de los grandes retos que debe asumir la FCCEA es la diversificación de sus opciones de grado, pues la concentración en los seminarios de grado, sin demeritar los procesos que ahí se desarrollan, ha restado espacios para los procesos de investigación, situación que se consolida como un indicador negativo, máxime en las actuales condiciones de acreditación de calidad de los programas académicos en Colombia.

Se debe empoderar a los maestros en el tema de investigación, pues son estos quienes pueden incidir, desde los inicios del proceso educativo en los estudiantes, para que construyan su opción de grado paralelamente con su avance académico; además, pueden generar nuevos imaginarios en torno a las opciones de grado, donde estas no sean vistas como un obstáculo para finalizar el proceso, sino, muy por el contrario, como un proyecto académico integral.

Finalmente, en términos institucionales, la universidad debe cualificar su política de investigación a fin de ser más atractiva para los integrantes de la comunidad académica. Además, se deben consolidar espacios de formación permanente que permitan la cualificación del talento humano al servicio de los procesos investigativos.

\section{Referencias}

Alfonso de Silvero, M. (2012). Retos del docente universitario del siglo XXI. En XII Coloquio Internacional de Gestión Universitaria. Universidad Veracruzana, Veracruz (México).

Alvarado, L. \& García. M. (2008, diciembre). Características más relevantes del paradigma socio-crítico: su aplicación en investigaciones de educación ambiental y de enseñanza de las ciencias realizadas en el Doctorado de Educación del Instituto Pedagógico de Caracas. Sápiens, 9(2), 187-202. Caracas: Universidad Pedagógica Experimental Libertador.

Antúnez, S. (1999). El trabajo en equipo de los profesores y profesoras: factor de calidad, necesidad y problema. El papel de los directivos escolares. Revista Educar, (24). España: Universidad Autónoma de Barcelona.

Asamblea Nacional Constituyente. (1991). Constitución Política de Colombia de 1991. Artículo 69. En Gaceta Constitucional. Bogotá. 
Barragán, D. \& Patiño, L. (2013). Elementos para la comprensión del fenómeno de la deserción universitaria en Colombia. Más allá de las mediciones. Cuadernos Latinoamericanos de Administración, 9(16). Bogotá: Universidad El Bosque.

Colombia, Alcaldía de Florencia. (2016). Plan de desarrollo 2016-2019. Florencia, Caquetá.

Congreso de la República de Colombia. (1982). Ley 60. Por la cual la Regional Florencia de la Universidad Surcolombiana, se transforma en la Universidad de la Amazonia. Diario Oficial 23.182, de enero 39 de 1982. Bogotá (DC).

Congreso de la República de Colombia. (1992). Ley 30. Por el cual se organiza el servicio público de la Educación Superior. Diario Oficial 40.700, de diciembre 29 de 1992. Bogotá (D. C.).

Consejo Académico Universidad de la Amazonia. (2009). Acuerdo 021 de 2009. Por el cual se reglamentan las Opciones de Grado de los estudiantes de pregrado de la Universidad de la Amazonia. Florencia, Caquetá.

Consejo Superior Universitario, Universidad de la Amazonia. (2007). Acuerdo 09 de 2007. Por el cual se adopta el Estatuto Estudiantil. Florencia, Caquetá.

Cornejo, R. (2009). Condiciones de trabajo y bienestar/ malestar docente en profesores de enseñanza media de Santiago de Chile. Educação \& Sociedade, 30(107). Brasil: Centro de Estudos Educação e Sociedade.

Corporación Universitaria Minuto de Dios. (2014). Acuerdo No. 04 Lineamientos para opciones de Pregrado. Recuperado de https://www.uniminuto.edu/ documents/941377/941434

División de Admisiones, Registro y Control Académico (DARCA). (2015). Informe de Gestión II-2015. Caquetá: Universidad de la Amazonia.

Gómez, C. (2015). Estrategias para el Fomento a la Educación Superior en Colombia: El Caso del Programa Jóvenes en Acción en la Universidad de la Amazonia. (Tesis de Especialización en Gestión Pública). Facultad de Posgrados, Escuela Superior de Administración Pública.

Ibáñez, C. (1994). Pedagogía y Psicología Interconductual. Revista Mexicana de Análisis de la Conducta, 20(1). Universidad Nacional Autónoma de México.

Maciel de Oliveira, C. (2005). La formación docente: mitos, problemas y realidades. En: Revista PRELAC, (1). Santiago de Chile: Oficina Regional de Educación de la Unesco para América Latina y el Caribe.

Mejía, C. (s. f.). Apuntes de un Investigador: Algo que debemos saber sobre educación, investigación y calidad en el marco de las nuevas políticas de desarrollo educativo en Colombia. Recuperado de http://www. colombiaaprende.edu.co/html/docentes/1596/ article-169771.html

Melo, L., Ramos, J., \& Hernández, P. (2014). La Educación Superior en Colombia: Situación Actual y Análisis de Eficiencia. Borradores de Economía, (808). Bogotá: Banco de la Republica de Colombia.

Ministerio de Educación Nacional. (2009). Líderes para el siglo XXI. Altablero, (50). Recuperado de http://www. mineducacion.gov.co/1621/article-195899.html

Ministerio de Educación Nacional. (mayo - junio de 2009). Maestros competentes. Altablero, (50). Recuperado de http://www.mineducacion.gov.co/1621/article-195576. html

Misas, G. (2004). La educación superior en Colombia: análisis y estrategias para su desarrollo. Bogotá: Universidad Nacional de Colombia.

Organización de las Naciones Unidas para la Educación, la Ciencia y la Cultura. (2015). Replantear la Educación ¿Hacia un bien común mundial? París: Ediciones Unesco.

Ospina, B. (2008). La educación como escenario para el desarrollo humano. Investigación y Educación en Enfermería, 26 (2). Medellín: Universidad de Antioquia.

Prieto, E. (2008). El papel del profesorado en la actualidad. Su función docente y social. Foro de Educación, (10). España: Universidad de la Rioja.

Restrepo, B. (s. f.). Conceptos y Aplicaciones de la Investigación Formativa, y Criterios para Evaluar la Investigación científica en sentido estricto. Recuperado de http://www.cna.gov.co/1741/articles-186502_doc_ academico5.pdf

Robalino, M. (2005). ¿Actor o Protagonista? Dilemas y responsabilidades sociales de la profesión docente.

Revista Prelac, (1). Santiago de Chile: Oficina Regional de Educación de la Unesco para América Latina y el Caribe. Ruiz, J. (2010). Importancia de la Investigación. Revista Científica. Venezuela: Universidad del Zulia. Recuperado 
de $\quad$ http://www.scielo.org.ve/scielo.php?script=sci_ arttext\&pid=S0798-22592010000200001\&lng=es\&tIng= es

Sierra, J. (2004). La investigación como prioridad universitaria. Revista virtual Universidad Católica del Norte. Medellín. Recuperado de http://revistavirtual. ucn.edu.co/index.php/RevistaUCN/article/view/285/540

Sierra, Z., Rojas, A. \& López, G. (2009). El Semillero de Investigación. Un Espacio de Vida. Orígenes y dinámica de los semilleros de investigación en Colombia: La Visión de los Fundadores. Cauca:Taller Editorial Universidad del Cauca.

Silva, A., Rodríguez, M., Gonzales, P. \& Sarmiento, J. (2008). Dinámicas de los Semilleros de Investigación en la UMNG. Revista de la Facultad de Ciencias Económicas, 16(1). Bogotá: Universidad Militar Nueva Granada.

Tamayo, M. (2002). El proceso dela investigación científica. Incluye evaluación y administración de proyectos de investigación. (4.a ed.). México, D.F.: Noriega Editores.
Tovar, J. \& Cárdenas, N. (2012). La importancia de la formación estratégica en la formación por competencias: evaluación de las estrategias de acción para la solución de problemas. Revista Electrónica de Investigación Educativa, 14(1), 122-135. Recuperado de http://redie. uabc.mx/vol14no1/contenido-tovarcardenas.html

Universidad de la Salle. (s. f.). Educación y Cultura. Recuperado de http://www.lasalle.edu.co/wps/wcm/ connect/31a678f3-f456-4cb9-b794-52913064aeae/ Educaci\%C3\%B3n+y+Cultura.pdf?MOD=AJPERES

Villegas, G. (s. f.). Reflexiones para una ley estatutaria de autonomía universitaria. III Foro sobre la reforma a la Educación Superior, Universidad del Rosario. Bogotá. 